\title{
Visual dynamics of a novel apparent movement effect ${ }^{1}$
}

\author{
M. S. MAYZNER and M. E. TRESSELT, \\ New York University, University Heights, \\ N.Y. 10453
}

By presenting the visual system with a highly complex spatio-temporal input configuration, a novel effect called "dynamic sequential displacement" has been disclosed. It is shown that the visual dynamics of this particular input configuration are unstable, yielding either (1) a coding of the input that loses all temporal ordering information or (2) a coding of the input that produces apparent movement and spatial displacement.

Until recently (Mayzner \& Tresselt, 1966), it has not been easy to present to the visual system highly complex spatio-temporal input configurations. However, by employing a computer-based cathode-ray tube (CRT) display system (Mayzner, Tresselt, \& Helfer, 1967a), it is now possible to display such complex configurations quite easily, and an increasing number of studies are employing such computer-based systems (Kahneman, 1967 ; Thorson, Lange, \& Biederman-Thorson, 1969; Uttal, 1969). With the use of this new display technology, previously unexplored visual phenomena, involving long strings of up to 100 sequentially displayed inputs at very high input rates (e.g., as fast as 50 microsec/input), have been studied, and novel effects, which we have called "sequential blanking" and "sequential displacement," have emerged (Mayzner, Tresselt, \& Helfer, 1967b). The present report examines a novel apparent movement effect combined with sequential displacement, which we shall refer to as "dynamic sequential displacement."

In an earlier study (Mayzner, Tresselt, Adrignolo, \& Cohen, 1967), we found that if the letters that constitute the word "somersault" are displayed sequentially in a given display order and at a particular display rate on the face of a CRT display, Ss observing such a display will report that, while they clearly perceive that all 10 letters of "somersault" are present, there appears to be a wide gap, a letter or two in width, between a particular adjacent pair of letters being displayed, when, in fact, all letters are equally spaced on the CRT surface. This effect we called "sequential displacement," since it represents a marked spatial displacement of certain inputs, which results directly from the spatio-temporal ordering of the input array, e.g., changing either input rate or input order will totally eliminate the effect. During the course of continuing investigations, designed in part to study sequential displacement effects further, a display sequence was uncovered that combines properties of both apparent movement and sequential displacement and that is characterized by several novel properties involving highly complex visual dynamics.

\section{METHOD}

A string of 20 adjacent characters or inputs was displayed sequentially across a CRT display surface, coated with an ultrashort persistence phospher (P24). A digital computer (Digital Equipment Corp. PDP-7) was employed to generate the display sequence on the CRT, which was interfaced to the computer. A complete description of this hardware system and its associated computer programs (software) may be found in earlier papers (Mayzner, 1968; Mayzner, Tresselt, \& Helfer, 1967a).

Table 1 gives the characters employed, the input display order used, and the input rate required to produce "dynamic sequential displacement." As may be seen, the characters involved sequences of "Os," "Os," and "-s," etc. The input display order is indicated by the row of numbers, where each number under the various characters indicates the order in which these five different character sequences were displayed. For example, with all five sequences of 20 characters, the first character at the left in the sequence was displayed first, the sixth character in from the left was displayed second, the 11th character in from the left was displayed third, etc. Each character was displayed for $5 \mathrm{msec}$, with a 5-msec pause between characters and a $5-\mathrm{msec}$ pause between the character displayed 20th and the character displayed first, since the display, once begun, was continuously cycled for a 120 -sec observation period.

Each character location occupied a cell, $1.25 \mathrm{~cm}$ high $\times 1.00 \mathrm{~cm}$ wide, and thus the horizontal extent of the total 20-character array was always $20 \mathrm{~cm}$. Each character had a display luminance of $1 \mathrm{~mL}$, as measured with a Gamma Scientific photometric system (Model 2020EA). S was positioned $100 \mathrm{~cm}$ from the display, with his head firmly positioned in a head and chin rest, and he was asked to maintain steady fixation on the center of the display throughout the observation period. Free viewing conditions produced essentially similar results to those found with steady fixation. Ss were dark-adapted for $5 \mathrm{~min}$ prior to the beginning of the several observation periods, and $10 \mathrm{Ss}$ were employed in the study. Without dark adaptation and a low level of background illumination, such as that provided by a tensor lamp, all effects remained the same. RESULTS AND DISCUSSION

Previous pilot work had shown that if the display sequences given in Table 1 were displayed at much faster input rates, e.g., $1 \mathrm{msec}$ "on," $1 \mathrm{msec}$ "off," and a 1-msec pause between cycles, all Ss reported clearly perceiving all 20 characters simultaneously, with a very slight flicker to the display, while at much slower input rates, e.g., $50 \mathrm{msec}$ "on," $50 \mathrm{msec}$ "off," and a 50-msec pause between cycles, all Ss reported clearly perceiving all characters, but clearly occurring sequentially and jumping from one display location to another on the display surface, in accordance with the display order employed. Only with an input rate of $5 \mathrm{msec}$ "on," $5 \mathrm{msec}$ "off," and a 5 -msec pause between cycles, or input rates very close to this rate, does "dynamic sequential displacement" occur. At this critical input rate, which it should be noted represents a total display time of $200 \mathrm{msec}$ to cycle the sequence just once and which corresponds to the critical time constant found previously to maximize sequential blanking and displacement effects (Mayzner,

Table 1

Various Input Contents and Input Display Order for a Continuously Cycling Display (“On” Time = 5 Msec, “Off” Time = 5 Msec, Cycle Pause = 5 Msec)

\begin{tabular}{|c|c|c|c|c|c|c|c|c|c|c|c|c|c|c|c|c|c|c|c|c|}
\hline \multirow{5}{*}{ Display Characters } & 0 & 0 & 0 & 0 & 0 & 0 & 0 & 0 & 0 & 0 & 0 & 0 & 0 & 0 & 0 & 0 & 0 & 0 & 0 & 0 \\
\hline & 0 & - & 0 & - & 0 & - & 0 & - & 0 & - & 0 & - & 0 & - & 0 & - & 0 & - & 0 & - \\
\hline & 0 & - & 1 & 0 & - & 1 & 0 & - & j & 0 & - & 1 & 0 & - & 1 & 0 & - & 1 & 0 & - \\
\hline & 0 & - & $i$ & $!$ & 0 & - & 1 & $!$ & 0 & - & 1 & $!$ & 0 & - & 1 & $!$ & 0 & - & 1 & $!$ \\
\hline & - & $!$ & - & $!$ & - & $!$ & - & $!$ & - & $!$ & - & $!$ & - & $!$ & - & $!$ & - & $!$ & - & $!$ \\
\hline Display Order & 1 & 5 & 9 & 13 & 17 & 2 & 6 & 10 & 14 & 18 & 3 & 7 & 11 & 15 & 19 & 4 & 8 & 12 & 16 & 20 \\
\hline
\end{tabular}


Tresselt, \& Helfer, 1967b), all $10 \mathrm{Ss}$ reported seeing one of two organizations, which reverse back and forth, seemingly spontaneously, as in Necker cube reversals, during the 120 -sec observation period employed with each of the five display sequences shown in Table 1.

In one organization, Ss report, for example, with a line of 20 "Os," a line of 20 "Os" with a slight flicker present, but which may suddenly change to a second organization of from only five to six "Os" spaced equally apart across the width of the display surface (i.e., $20 \mathrm{~cm}$ ) and moving from left to right, just as "ducks in a shooting gallery." If, for the 20 "Os," we substitute any one of the other content lines shown in Table 1 , all $10 \mathrm{Ss}$ again report seeing either all 20 characters with a slight flicker or seeing a sudden change to the "ducks in the shooting gallery" type of movement, but with the different content inputs (i.e., "O-" or "O- $/$," etc.) that are moving from left to right most remarkably all occupying the identical spatial location. Thus, with the input array "O-/!," all Ss report that it appears as if the letter " $O$ " had a dash, a slash, and an exclamation point inside it. The dash and exclamation point alone appears like a plus sign moving across the display surface. These novel effects, including both apparent movement and spatial displacement, we have called "dynamic sequential displacement."

Each of the 10 Ss observed each of the five different display sequences five times each in a different random order and were asked to report, for each 120-sec observation period, each time the organization of the display sequence changed from 20 characters to 5 or 6 characters in movement, or back again. Time spent in observing each organization also was recorded. Since all Ss were quite consistent across the five different time observation periods used on each of the five different display sequences, the results for all observation periods were combined and averaged. The mean number of reversals per observation period was 4.7 , with a range of from 2 to 11 . Time spent per organization was $51.3 \mathrm{sec}$ on the average for the 20 characters and $68.7 \mathrm{sec}$ on the average for 5 or 6 characters in movement, per observation period. However, the critical finding of the study is not the actual number of reversals that occur or time spent per organization, but rather that two entirely different processings or organizations of the input array are possible and that the visual dynamics of the array are sufficiently unstable so that the system oscillates between these two different organizational states.

One organization loses all temporal ordering information and codes the input array as if all inputs had occurred simultaneously. The other organization, which occurs equally often, codes the input array in such a way that (1) the visual system is detecting only what appears to be 5 or 6 spatially separate inputs rather than 20 inputs, but in apparent movement, and (2) when input content differs, such as "Os," "-s," "/s," and $! s$ " interleaved, these inputs suffer a spatial displacement such that all four inputs appear to be occupying the identical physical location in space, when, in fact, all four inputs are spatially adjacent to one another in space.

Obviously, very complicated visual dynamics must be occurring when the visual system is required to process such a highly complex spatio-temporal input configuration. ${ }^{2}$ In other papers (Mayzner \& Tresselt, in press; Mayzner, Tresselt, \& Helfer, 1967b), we have attempted to construct a visual information-processing model based on excitatory-inhibitory field interactions designed to handle certain sequential blanking and displacement effects, but the "dynamic sequential displacement" effect reported here must await further development of our model before it can begin to account satisfactorily for the present rather complex and novel findings.

\section{REFERENCES}

KAHNEMAN, D. An onset-onset law for one case of apparent motion and metacontrast. Perception \& Psychophysics, 1967, 2, 577-584.

MAYZNER, M. S. The research potential of a computer-based cathode-ray tube display system. Behavior Research Methods \& Instrumentation, 1968, 1, 41-43.

MAYZNER, M. S., \& TRESSELT, M. E. Studies in sequential perception: Looking at one thing but finding another. Perceptual \& Motor Skills, $1966,23,257-258$.

MAYZNER, M. S., \& TRESSELT, M. E. Visual information processing with sequential inputs: A general model for sequential blanking, displacement, and overprinting phenomena. Annals of The New York Academy of Sciences, in press.

MAYZNER, M. S., TRESSELT, M. E., \& HELFER, M. S. A research strategy for studying certain effects of very fast sequential input rates on the visual system. Psychonomic Monograph Supplements, 1967a, 2(5, Whole No. 21), 73-81.

MAYZNER, M. S., TRESSELT, M. E., \& HELFER, M. S. A provisional model of visual information processing with sequential inputs. Psychonomic Monograph Supplements, 1967b, 2(7, Whole No. 23), 91-108.

MAYZNER, M. S. TRESSELT, M. E., ADRIGNOLO, A. J., \& COHEN, A. Further preliminary findings on some effects of very fast sequential input rates on perception. Psychonomic Science, 1967, 7, 281-282.

THORSON, J., LANGE, G. D., \& BIEDERMAN-THORSON, M. Objective measure of the dynamics of a visual movement illusion. Science, $1969,164,1087-1088$.

UTTAL, W. R. Masking of alphabetic character recognition by dynamic visual noise (DVN). Perception \& Psychophysics, 1969, 6, 121-128.

\section{NOTES}

1. This research was supported by Grant No. GB-8037 from the National Science Foundation to the first author.

2. Eye movements would not seem to be involved in "dynamic sequential displacement," since a control display was constructed, which consisted of two lines of characters, as those shown in Table 1, one line directly below the other, but with reversed display orders in the two lines. With such a display, all Ss again report "dynamic sequential displacement," but with movement from left to right in the top line and the opposite movement, i.e., from right to left, in the bottom line, as would be expected if the effect is central in origin.

\section{Transfer effects of an observation stimulus as a function of its relevancy}

STANFORD H. SIMON, Veterans
Administration Center, Wood, Wis. 53193

Three groups of Ss, 20 each, learned three successive concept identification (CI) tasks, on which one group had no observation stimulus ( $S^{\circ}$ ), a second group had a relevant $S^{\circ}$, and a third group had an irrelevant $S^{\circ}$. Each type of $S^{\circ}$ continued to affect CI learning as a function of its relevancy. Predictions of relative improved performance on later tasks by the irrelevant $S^{o}$ group, based on a rational operator model, did not occur. 